\title{
Is your error my concern? An event-related potential study on own and observed error detection in cooperation and competition
}

\author{
Ellen R. A. de Bruijn ${ }^{1,2}$ * and DanielT. von Rhein ${ }^{2}$ \\ 1 Unit of Clinical Psychology, Institute of Psychology, Leiden University, Leiden Institute for Brain and Cognition, Leiden, Netherlands \\ 2 Donders Institute for Brain, Cognition and Behaviour, Radboud University Nijmegen, Nijmegen, Netherlands
}

Edited by:

Eldad Yechiam, Technion Israel

Institute of Technology, Israel

\section{Reviewed by:}

Michal Lavidor, Bar Ilan University, Israel

Guy Hochman, Duke University, USA

*Correspondence:

Ellen R. A. de Bruijn, Institute of Psychology, Leiden University, Pieter de la Courtgebouw, Wassenaarseweg 52, 2333 AK Leiden, Netherlands. e-mail: edebruijn@fsw.leidenuniv.nl
Electroencephalogram studies have identified an error-related event-related potential (ERP) component known as the error-related negativity or ERN, thought to result from the detection of a loss of reward during performance monitoring. However, as own errors are always associated with a loss of reward, disentangling whether the ERN is error- or reward-dependent has proven to be a difficult endeavor. Recently, an ERN has also been demonstrated following the observation of other's errors. Importantly, other people's errors can be associated with loss or gain depending on the cooperative or competitive context in which they are made. The aim of the current ERP study was to disentangle the error- or reward-dependency of performance monitoring. Twelve pairs $(N=24)$ of participants performed and observed a speeded-choice-reaction task in two contexts. Own errors were always associated with a loss of reward. Observed errors in the cooperative context also yielded a loss of reward, but observed errors in the competitive context resulted in a gain. The results showed that the ERN was present following all types of errors independent of who made the error and the outcome of the action. Consequently, the current study demonstrates that performance monitoring as reflected by the ERN is error-specific and not directly dependent on reward.

Keywords: performance monitoring, response ERN, observed ERN, cooperation, competition, reward

\section{INTRODUCTION}

Human behavior is prone to errors. We slip, trip, push incorrect buttons, and drop things on a regular basis. Fortunately, people are in general able to detect these errors in a fast and efficient manner. This fast error detection process is enabled by a continuous monitoring of our performance and thus importantly facilitates goal-directed behavior. Electroencephalogram (EEG) studies have identified an event-related potential (ERP) component immediately following own errors known as the errorrelated negativity or ERN (Falkenstein et al., 1990; Gehring et al., 1993). The ERN is elicited $50-70 \mathrm{~ms}$ following an erroneous button press and is thought to originate from areas in posterior medial frontal cortex (pMFC), including anterior cingulate cortex (ACC) and pre-supplementary motor area (pre-SMA; Holroyd et al., 2004; Ridderinkhof et al., 2004; De Bruijn et al., 2009). Research and theories on the ERN and performance monitoring have proposed a close link between error and reward processing. The goal of the current study is to disentangle the error- or reward-dependency of performance monitoring using ERPs.

One of the most influential theories on the ERN in performance monitoring is the reinforcement learning (RL) theory proposed by Holroyd and Coles (2002). The ERN can be elicited immediately following response onset, the so-called response ERN or rERN or following negative feedback, the so-called feedback related negativity or FRN. The RL theory proposes that depending on the knowledge available to the system, the ERN is elicited at the first moment in time the error can be detected. Importantly, this theory proposes a direct relation between performance monitoring or error detection and reward processing. In short, the RL theory states that whenever an error is made, the outcome of an action turns out to be worse than expected, resulting in a loss of reward and hence eliciting the ERN. However, as own errors are always negative events associated with some loss of reward, disentangling whether the ERN is error- or reward-dependent has proven to be an extremely difficult endeavor.

Humans are social animals and for a large part of the day involved in some form of social interaction. Experience teaches us that people are not only able to detect their own errors, but that they are also extremely skilled in detecting other people's mistakes. However, for long, research focused on performance monitoring in individual settings, thus only reporting findings on monitoring of our own performance.

More recently, performance monitoring research has expanded into more social settings and an ERN, the so-called observed ERN (oERN) has also been demonstrated following the observation of other's errors (Miltner et al., 2004; van Schie et al., 2004; Bates et al., 2005). These initial ERP studies suggested that similar neural processes are responsible for the detection of one's own errors as well as the detection of other's errors. This assumption was later confirmed in fMRI studies showing that indeed overlapping areas in PMFC were stronger activated for own and observed errors in 
comparison to correct performance and observations (Shane et al., 2008; De Bruijn et al., 2009).

Importantly, depending on whether the observed errors are made in cooperative or competitive contexts they are associated with loss or gain for the observer, respectively. Thus, we realized that by using the overlapping mechanisms for observed and own error detection we had a method to disentangle the error- or reward-dependency of performance monitoring. For this aim, we recently conducted an fMRI study in which we directly compared monitoring of own and other's performance in both a cooperative and a competitive setting (De Bruijn et al., 2009). As in real-life, own errors were associated with a loss of money in both settings, while observed errors also resulted in a loss of money in the cooperative setting, but led to a monetary gain in the competitive context. The results from this study demonstrated that the areas in pMFC involved in detecting own errors were similarly involved in detecting other's errors independent of the award associated with the cooperative or competitive context, thus demonstrating the errorspecificity of these areas. On the other hand, areas known to be involved in reward processing, like ventral striatum, were sensitive to the nature of the context and responded in a reward-specific manner.

Although some recent studies have investigated ERP correlates of observed performance monitoring in cooperative and competitive contexts, the focus so far has mainly been on the FRN elicited by negative feedback. Two recent studies demonstrated increased FRN amplitudes in a cooperative compared to a competitive setting, suggesting that the FRN in these tasks were more sensitive to the loss of reward associated with the outcome (Itagaki and Katayama, 2008; Marco-Pallares et al., 2010). Importantly, however, a focus on the FRN has some consequences for the analyses and the choice of paradigm. First of all, unlike the response-locked oERN, it is difficult to fully dissociate between the FRN and the following stimulus-locked P300 ERP component as both are involved in processing unexpected, but task-relevant events (see, e.g., Hajcak et al., 2005). This is especially the case when the FRN is analyzed at a more central electrode like Cz. Second, the focus on the FRN has important consequences for the paradigms used. Speededchoice-reaction tasks are mainly used in rERN and oERN tasks to elicit a reasonable amount of errors. Studies aimed at investigating the FRN, however, usually employ gambling tasks in which participants have little or no control over the correctness of their responses and thus need to fully rely on the feedback information. So although it is generally assumed that the FRN and ERN are reflections of the same performance monitoring process (see, e.g., Holroyd et al., 2004; Mars et al., 2005), important differences in quantification and experimental design exist that may importantly affect the exact outcomes of these observed error studies.

To our knowledge, only one recent study did investigate observed errors relative to the moment of the actor's response in a cooperative and competitive context. The results from this go/nogo study by Koban et al. (2010) were more in line with our recent fMRI findings, by demonstrating an oERN of similar amplitude for observed errors in both contexts. The study did demonstrate a latency difference, with the oERN peaking later in the competitive setting. However, when directly comparing the ERP waveforms from the original observed error monitoring study by van Schie et al. (2004) and those in the study by Koban et al. large differences become apparent. Importantly, the waveforms in the Koban et al. may alternatively be interpreted as resembling stimulus-locked ERPs more than response-locked ERPs. These differences in ERP waveforms raise the question whether the exact same processes are investigated in the two paradigms.

In the observed Flanker paradigm in the van Schie et al. (2004) study participants were seated facing each other and observed the actor's actual thumb movements, which were taken as the moment of response onset. In the Koban et al. (2010) study, participants were seated next to each other, looking at a computer screen and inferred the correctness of the actor's response on the basis of a visual stimulus presented on the screen (i.e., a black frame appearing around the target stimulus indicating that a response had been given). So although ERPs were in theory time-locked to the moment of the actor's response, this time-point was visualized for the observer by the presentation of a stimulus. The observer thus had to process this abstract visual stimulus and then infer the correctness of the given response. It is plausible that this explains why the ERP waveforms from the observation condition in the Koban et al. study differ from the initial oERN study by van Schie et al. as the presentation and subsequent processing of the visual stimulus may result in a stimulus-locked ERP pattern. Consequently, it is rather difficult to determine whether the negativities analyzed in the Koban et al. paper are superimposed on response-locked or stimulus/feedback-locked error-related ERPs.

An important explanation for the presence of the oERN in the van Schie et al. (2004) study was based on sub-threshold simulation of the goal movement and comparing this to the actual observed movement. This was supported by dedicated analyses investigating the relative activation of the left or right motor cortex during observation. These so-called lateralized readiness potential (LRP) analyses demonstrated covert lateralized activation of the motor cortex. In other words, while observing, participants activated the correct response at a sub-threshold level even before the actor started responding and thus generated a representation of the appropriate response following stimulus presentation. However, when they subsequently observed an error from the actor this initial correct activation was inhibited and the observers showed increased activation of the incorrect response. So, following the response of the other participant, the observer's motor system was differentially activated as a function of the accuracy of the observed response. This covert motor simulation may thus play a central role in the mechanisms underlying observed error detection. Another advantage of the setup by van Schie et al. was that it represented a situation which was more comparable to real-life performance monitoring in a social context, as the relation between the observed erroneous movement and the process of performance monitoring was very direct. This relation was more indirect in the Koban et al. (2010) study, as the error detection process was mediated by the presentation of a more abstract visual stimulus. As a result, the motor simulation processes underlying observed performance monitoring might have been weakened in the later study. In our view, the existing differences in paradigms used and the processes involved as well as the forthcoming uncertainties importantly warrant investigating the error- or reward-dependency of the ERN using the observed flankers paradigm. 
The aim of the current study was to disentangle the erroror reward-dependency of performance monitoring using ERPs. This was achieved by investigating the ERN following own and observed errors in a cooperative and competitive context using the observed Flankers paradigm. Based on our previous fMRI study (De Bruijn et al., 2009) and the results by Koban et al. (2010) we expected to find similar rERN and oERN amplitudes in both contexts, reflecting the error-dependency of this ERP component.

\section{MATERIALS AND METHODS PARTICIPANTS}

Twelve pairs of participants $(N=24 ; 14$ women; mean age $=23.1$ years, $\mathrm{SD}=2.9$ ) participated in the experiment. All subjects were healthy volunteers recruited on the campus of the Radboud University in Nijmegen, the Netherlands and were either paid or awarded course credits for participation. All participants gave their informed consent after the nature of the study had been fully explained to them. The study was carried out in accordance with the latest version of the Helsinki Declaration and was approved by the local ethics committee of the university.

\section{DESIGN AND PROCEDURE}

The experimental setup can be seen in the left panel of Figure $\mathbf{1}$. Two participants were scheduled for one meeting and randomly assigned to start the task as an actor or observer. They were seated face-to-face at a table. In the middle of the table, between the two participants, a wooden box with a LED display was placed. The actor responded with joystick movements in one horizontal axis. The stick had a central position and moved back to this position when the force was removed. The joysticks were positioned, such that (1) the actor could easily reach the sticks with his/her thumbs and that (2) the observer was able to see the joysticks and thumbs well. On the actors' side of the LED display, arrow stimuli from a modified Flankers task were presented (Eriksen and Eriksen, 1974). On the observers' side of the display, only the target arrow was presented, indicating which joystick had to be moved.

Stimulus presentation was controlled with Presentation software (Neurobehavioral Systems). Each trial began with the presentation of a fixation circle $(300 \mathrm{~ms})$, followed by an empty display (300 ms). Next, the stimulus was presented for $300 \mathrm{~ms}$. All stimuli consisted of five arrows. The central target arrow indicated the goal direction of the actor's movement. The two flanking arrows on each side could either be congruent or incongruent with the target. Following the stimulus, an empty display was presented for $900 \mathrm{~ms}$.

Actors were instructed to touch the two joysticks with their thumbs and respond to the target arrow as fast as possible. When the central arrow on the display pointed to the right, they had to press the right stick with their right thumb to the right. Analogously, they were required to press the left stick with their left thumb to the left, when the central arrow pointed to the left.

Observers were instructed to count the errors made by the actor (see van Schie et al., 2004). They were told that the initial response counted and that any corrective behavior of an error was invalid. They were asked to keep the amount of errors in mind and write it down during the break between the blocks. After the first condition, a break was held, and participants switched their tasks.

The task was performed in both a cooperative and a competitive context. The order of these two contexts was counterbalanced between pairs. Participants started the cooperative context with

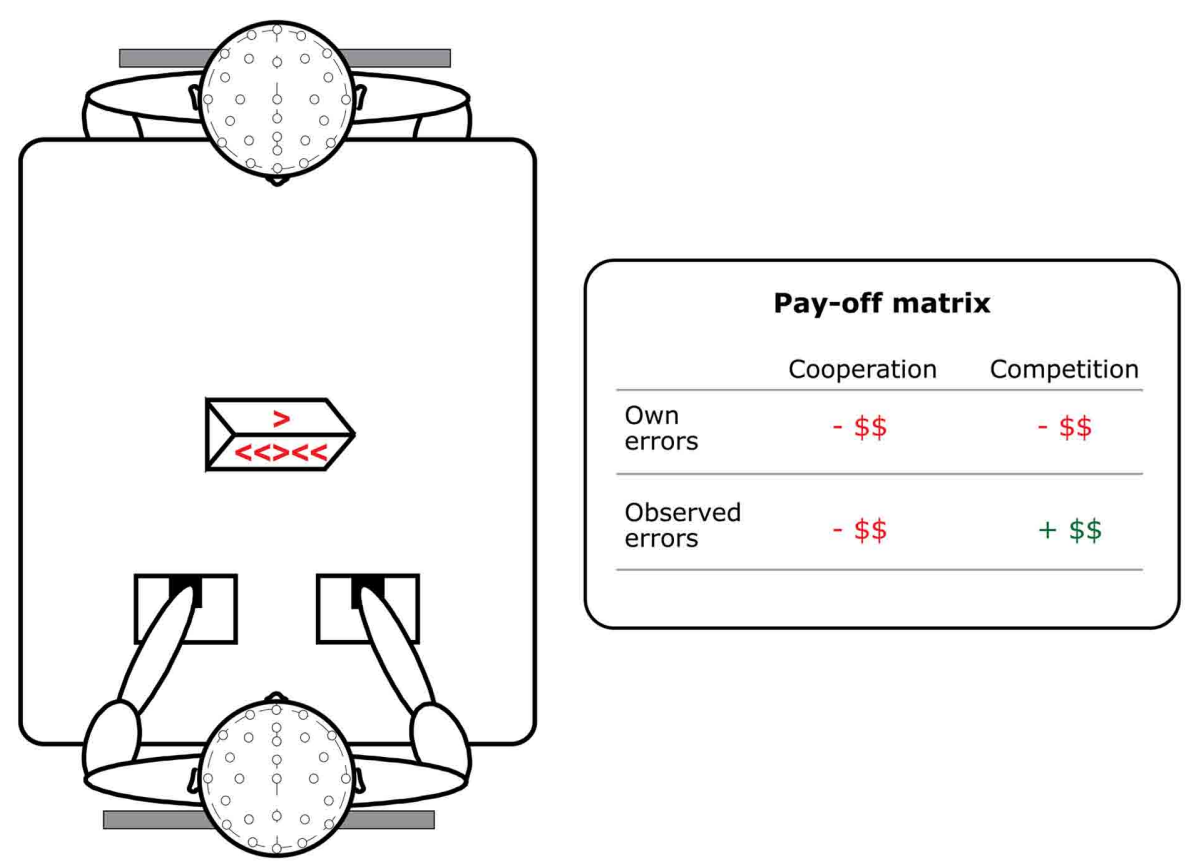

FIGURE 1 | Setup of the observed Flankers task. Participants both performed and observed 600 trials in a cooperative and in a competitive context. Own errors were always associated with a loss of money. Observed errors in the cooperative context also resulted in a loss of reward, but observed errors in the competitive context resulted in a gain. 
a shared bonus of 10 Euros, but for each error 10 cents were deducted from this bonus, irrespective of whether a participant made the error or whether they observed their partner make an error (see Figure 1, right panel and De Bruijn et al., 2009). In the competitive context each participant started out with an individual bonus of five Euros. Again 10 cents were deducted when they made an error themselves, but they would gain 10 cents when their opponent made an error.

Participants performed and observed six blocks of 100 trials in each context. As a result, the entire experiment consisted of 2400 experimental trials and 160 test trials (40 before each new condition). Congruent and incongruent stimuli were presented in a random order and equally distributed in each block (each stimulus type was presented 150 times in each context). After each block, there was a break of approximately $1 \mathrm{~min}$.

\section{DATA ACQUISITION AND ANALYSES}

The EEG was recorded from the two participants using 27 tin electrodes mounted in an elastic electrode cap (Electrocap International). Electrodes were placed at 7 midline $(\mathrm{FPz} / \mathrm{AFz} / \mathrm{Fz} / \mathrm{FCz} / \mathrm{Cz} / \mathrm{Pz} / \mathrm{Oz})$ and 20 lateral $(\mathrm{FP} 1-2 / \mathrm{F} 7-$ 8/F3-4/FC5-6/T3-4/C3-4/CP5-6/T5-6/P3-4/O1-2) locations in accordance with an extension of the international 10-20 system. All signals were referenced to the left mastoid, but later re-referenced to the average of both mastoids. The vertical electrooculogram (EOG) was recorded bipolarly from electrodes placed above and below the right eye. The horizontal EOG was also recorded bipolarly from electrodes lateral to both eyes. All electrode impedances were kept below $5 \mathrm{k} \Omega$. The EEG and EOG signals were amplified using a time constant of $8 \mathrm{~s}$ (high pass $0.02 \mathrm{~Hz}$ ) and were filtered off-line with a bandpass of 1-14 Hz. All signals were digitized with a sampling rate of $500 \mathrm{~Hz}$.

Trials with response times faster than $150 \mathrm{~ms}$ were excluded from all analyses (1.5\%). For the behavioral analyses, we analyzed performance and response times of the actors by entering individual mean error rates and reaction times in a repeated measures general linear model (GLM) with Context (cooperative vs. competitive), Congruency (congruent vs. incongruent), and Correctness (correct vs. incorrect) as possible within-subject factors.

Before averaging EEG signals to ERPs, error and correct trials were matched for reaction times ( $\pm 4 \mathrm{~ms}$; see van Schie et al., 2004) to control for possible differential effects of the stimuluslocked ERP components. Eye movements were corrected using the procedure described by Gratton et al. (1983) and averaged to ERPs separately for each subject and each condition, relative to a $100-\mathrm{ms}$ pre-response baseline. Note that a peak measure implies the presence of a negative peak on correct ERP waveforms as well. However, inspection of our individual averages revealed that this was often not the case for both the actor and observer data. Therefore, we conducted ERN analyses in which the ERN was quantified as a mean amplitude measure. Based on the grand averages, we defined the rERN as the mean amplitude of the interval from 0 to $150 \mathrm{~ms}$ after response onset and the oERN as the mean amplitude in the 150- to 300-ms time window after the response. Individual rERN and oERN amplitudes were entered into $2 \times 2$ repeated measures GLMs with
Context (cooperative vs. competitive) and Correctness (correct vs. incorrect) as within-subject factors. Finally, latencies of the rERN and oERN peaks on incorrect responses were analyzed by entering the individual latencies into repeated measures GLMs with Context (cooperative vs. competitive) as within-subject factor.

\section{RESULTS}

\section{BEHAVIORAL ANALYSES}

As expected, the analyses on error rates demonstrated a main effect of Congruency, indicating increased error rates for incongruent trials $(22.2 \%)$ than for congruent ones $[6.7 \% ; F(1,23)=124.08$, $p<0.001]$. There was neither a main effect of Context present $[F(1,23)=1.85, p=0.186]$, nor a significant interaction between Congruency and Context $(F<1)$.

When investigating reaction times for correct responses only, we found the expected main effect for Congruency, with slower reaction times for responses to incongruent stimuli (352 ms) compared to responses to congruent stimuli $[312 \mathrm{~ms} ; F(1,23)=97.05$, $p<0.001]$. Neither the main effect of Context, nor the interaction between the two was significant (both $F s<1$ ). When incorrect responses were also included in the analysis, an additional effect of Correctness was found, reflecting the usually found faster reaction times for erroneous responses $(273 \mathrm{~ms})$ compared to correct ones [332 ms; $F(1,23)=273.37, p<0.001]$.

\section{ACTOR ERP ANALYSES (rERN)}

The ERP waveforms for the actor in the performance condition are depicted in Figure 2. The well-known rERN component is visible following incorrect responses at midline electrodes with the expected frontocentral distribution (Figure 3, upper panel) peaking around $70 \mathrm{~ms}$. The peak latencies were not different for the two contexts $(F<1)$.

As expected, the amplitude analyses demonstrated a main effect of Correctness, with more negative amplitudes for incorrect responses $(-2.76 \mu \mathrm{V})$ compared to correct ones $[3.08 \mu \mathrm{V}$; $F(1,23)=75.25, p<0.001]$. Neither the main effect of Context $[F(1,23)=2.66, p=0.117]$, nor the interaction between Correctness and Context $(F<1)$ reached significance, indicating that rERN amplitudes did not differ between the two contexts.

\section{OBSERVER ERP ANALYSES (oERN)}

The ERP waveforms for the observer in the observation condition are depicted in Figure 4. The oERN component is visible following incorrect responses at the midline electrodes and also shows the typical frontocentral distribution (Figure 3, lower panel). The oERN peak latencies did not differ between the two contexts $(F<1$; cooperative $=232 \mathrm{~ms}$; competitive $=237 \mathrm{~ms})$.

Similar to the rERN outcomes, the amplitude analyses demonstrated a main effect of Correctness, with more negative amplitudes for incorrect responses $(-2.75 \mu \mathrm{V})$ compared to correct ones $[-0.74 \mu \mathrm{V} ; F(1,23)=43.71, p<0.001]$. Neither the main effect of Context $(F<1)$, nor the interaction between Correctness and Context $[F(1,23)=2.32, p=0.141]$ reached significance, indicating that oERN amplitudes did not differ between the two contexts. 


\section{Performance}

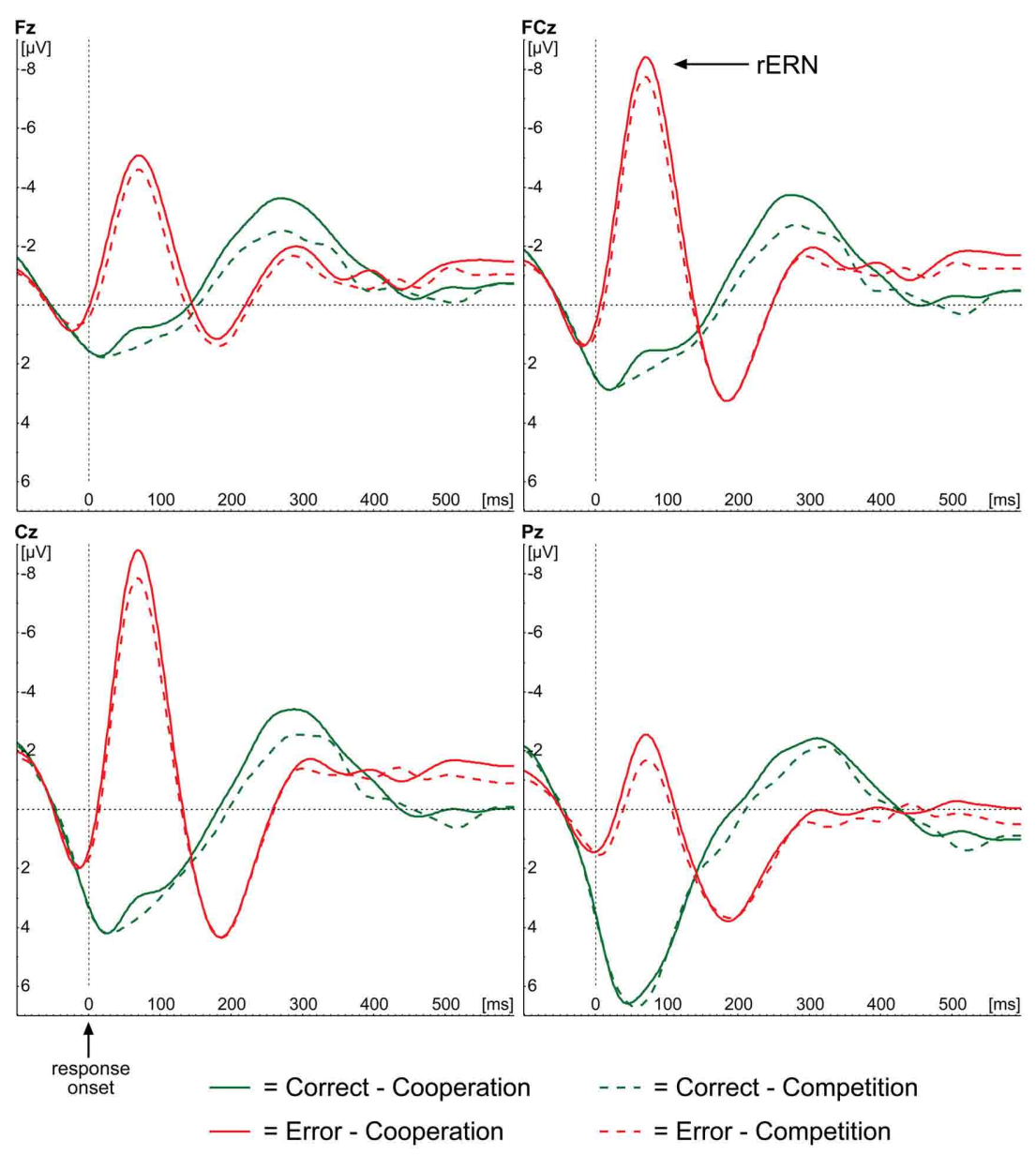

FIGURE 2 | Grand average ERP waveforms for the performance condition, time-locked to the onset of the actor's response for correct (green lines) and incorrect (red lines) responses separately. Midline electrodes $\mathrm{Fz}, \mathrm{FC} z, \mathrm{Cz}$, and $\mathrm{Pz}$ are depicted.

\section{DISCUSSION}

The current study aimed at determining the error- or rewarddependency of performance monitoring by examining responselocked own and observed errors in a cooperative and competitive setting. The setup was designed with the aim to resemble real-life interactive behavior, by enabling EEG onsets to be time-locked to the actual actor's movement onset in a direct and comparable manner both for own and observed actions.

The results demonstrated the well-known rERN following own errors in the performance condition. The amplitude and latency of the rERN was comparable for both contexts. Moreover, a clear oERN was present following other's errors in the observe condition. The ERP waveform patterns for the observed actions time-locked to movement onset were very similar to those originally reported by van Schie et al. (2004), thus supporting our assumption that we are currently investigating the same processes and mechanisms. Importantly, the current study showed that the amplitudes and the latencies of the oERN did not differ between the cooperative and competitive context. As the reward-dependency was crucially manipulated for the observed errors (loss vs. gain) in the two contexts, our results are in favor of an error-specific explanation of the ERN. We do not find evidence supporting the reward-specificity of performance monitoring as suggested by the RL theory (Holroyd and Coles, 2002).

Notably, the current outcomes are in line with a previous fMRI study from our lab, which also revealed error-dependent activations in anterior cingulate and pre-SMA for own and observed errors in both contexts (De Bruijn et al., 2009). Previous studies have repeatedly demonstrated the source of the ERN to originate from areas in pMFC, including anterior cingulate (see, e.g., Ridderinkhof et al., 2004; Debener et al., 2005). Reward-dependent activations were found in the previous fMRI study in striatal areas including nucleus accumbens. Please note that, to enable a more direct comparison, the payoff matrix for errors in the two contexts used in the fMRI study was the same as the one currently used. Obviously, it is very well plausible that the reward-related activations are not reflected in the scalp-recorded ERN waveforms. Furthermore, the current results are in line with a recent study by 


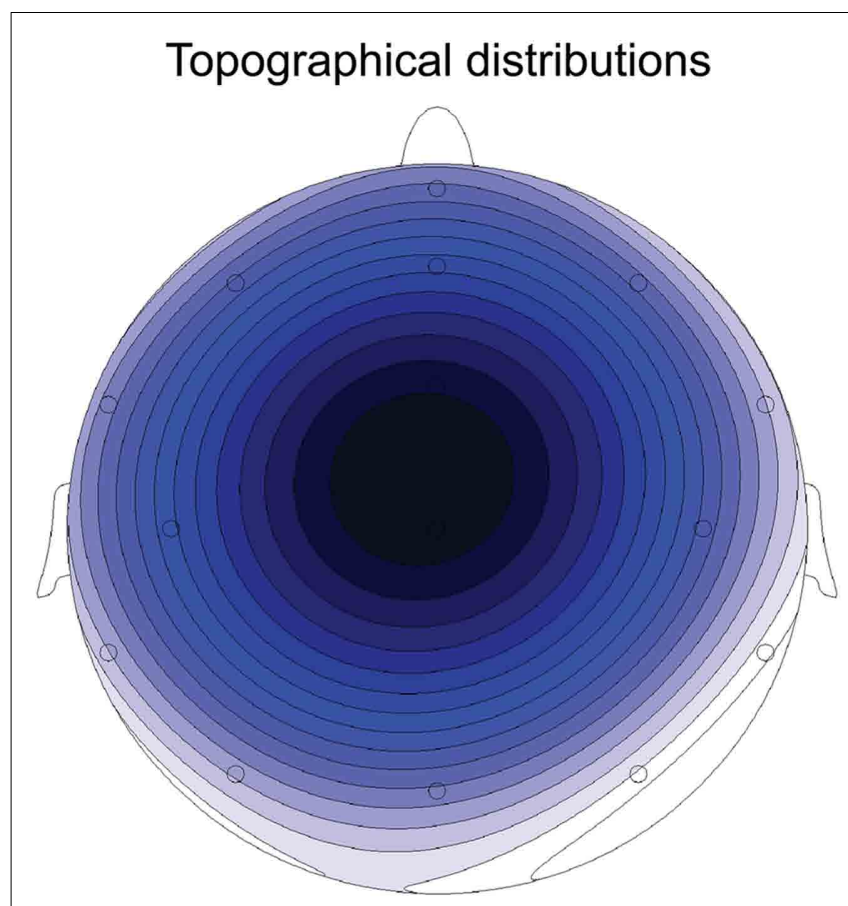

Performance - rERN (70 ms)

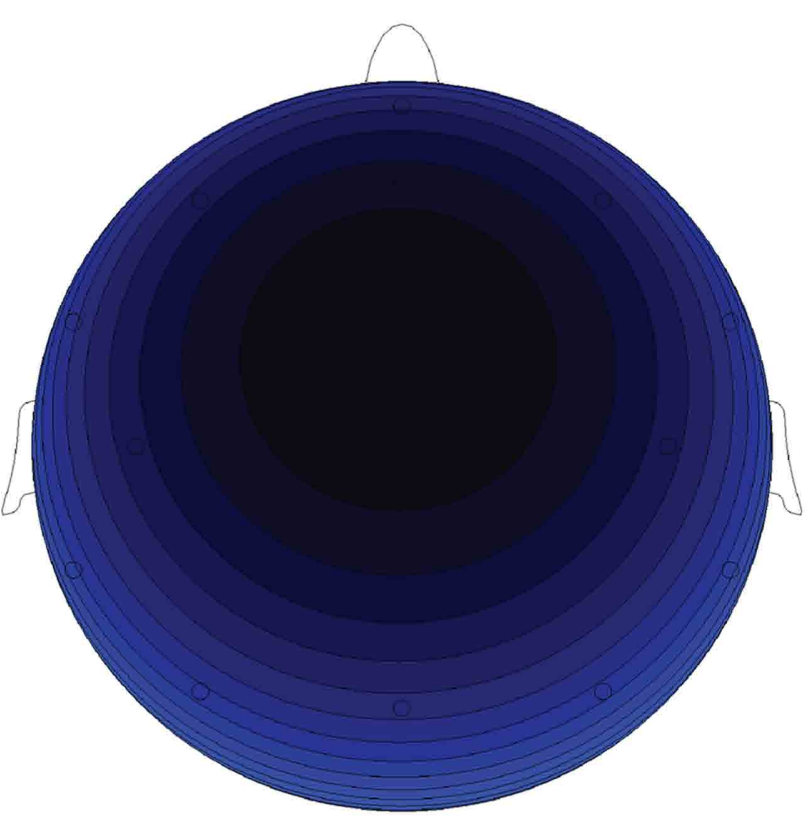

Observation - oERN (235 ms)

FIGURE 3 | Topographical distribution of the difference waves (incorrect minus correct) showing the expected frontocentral distribution for the rERN at peak onset $(70 \mathrm{~ms}$ ) in the Performance condition (upper panel) and for the oERN at peak onset $(235 \mathrm{~ms})$ in the Observation condition (lower panel). Darker colors indicate more negative amplitudes.

Koban et al. (2010), which also demonstrated similar amplitudes for observed errors in a cooperative and competitive context. The results from that study did however demonstrate a latency difference between the two contexts, with a later oERN peak in the competitive compared to the cooperative context. This finding was not present in the current data. Given the differences in methodology and resulting ERP waveforms between the current study and the one by Koban et al. it is difficult to draw conclusions regarding this aspect. It is, for example, not entirely clear whether the two paradigms measure the exact same processes.

To our knowledge, no other studies investigated the contextsensitivity of the oERN measured relative to response onset. Two recent studies did investigate the feedback-locked FRN in comparable cooperative and competitive settings (Itagaki and Katayama, 2008; Marco-Pallares et al., 2010). The current findings are not in line with these recent studies that demonstrated the FRN to be sensitive to the actual reward outcome. Although it is generally assumed that the rERN and the FRN are reflections of the same performance monitoring process (see, e.g., Holroyd et al., 2004; Mars et al., 2005), these divergent outcomes might suggest that the feedback-locked and response-locked ERNs actually reflect different processes in social performance monitoring. However, based on currently available evidence, we would like to refrain from concluding this. Importantly, experimental paradigms used in rERN and FRN studies are very different and thus the exact performance monitoring processes needed to perform the task may crucially differ. The studies by Itagaki and Katayama and MarcoPallares et al. made use of gambling tasks to investigate the FRN during social performance monitoring. Importantly however, performing a gambling task does not require internal performance monitoring, as participants do not have a representation of the correct response at the moment of response onset. Participants need to fully rely on the external feedback information to determine the correctness of their response and have no control over the outcome. Moreover, in complete gambling tasks, participants cannot optimize their behavior in any way, as associated feedbacks are predetermined by a computer program and thus each trial requires a new gamble.

Recent theories have proposed pMFC to be implicated in regulating adaptive behavior rather than performance monitoring alone. Rushworth and Behrens (2008) proposed pMFC to be crucially involved in updating of action values, optimizing performance, and subsequent strategy changes on the basis of reward information. Support for this more general role comes from studies that demonstrated amongst others pMFC to be similarly involved in processing correct actions associated with a high predictive value, as is the case in the beginning of learning (Walton et al., 2004). It also explains why pMFC is often found to be activated by other signals besides errors that also trigger the need for a behavioral change, like response conflict (Botvinick et al., 2004), error likelihood (Brown and Braver, 2005), or pain (Eisenberger and Lieberman, 2004; Singer et al., 2004). So, if indeed the central role of pMFC and associated ERP components is regulation of adaptive behavior, the exact function of the FRN elicited in tasks lacking behavioral control may thus be open for investigation.

A recent study by Holroyd et al. (2008) recently investigated this issue, by focusing on positive and negative ERP components elicited by different types of stimuli and feedback. Crucially, the authors concluded that the FRN is actually the same component 


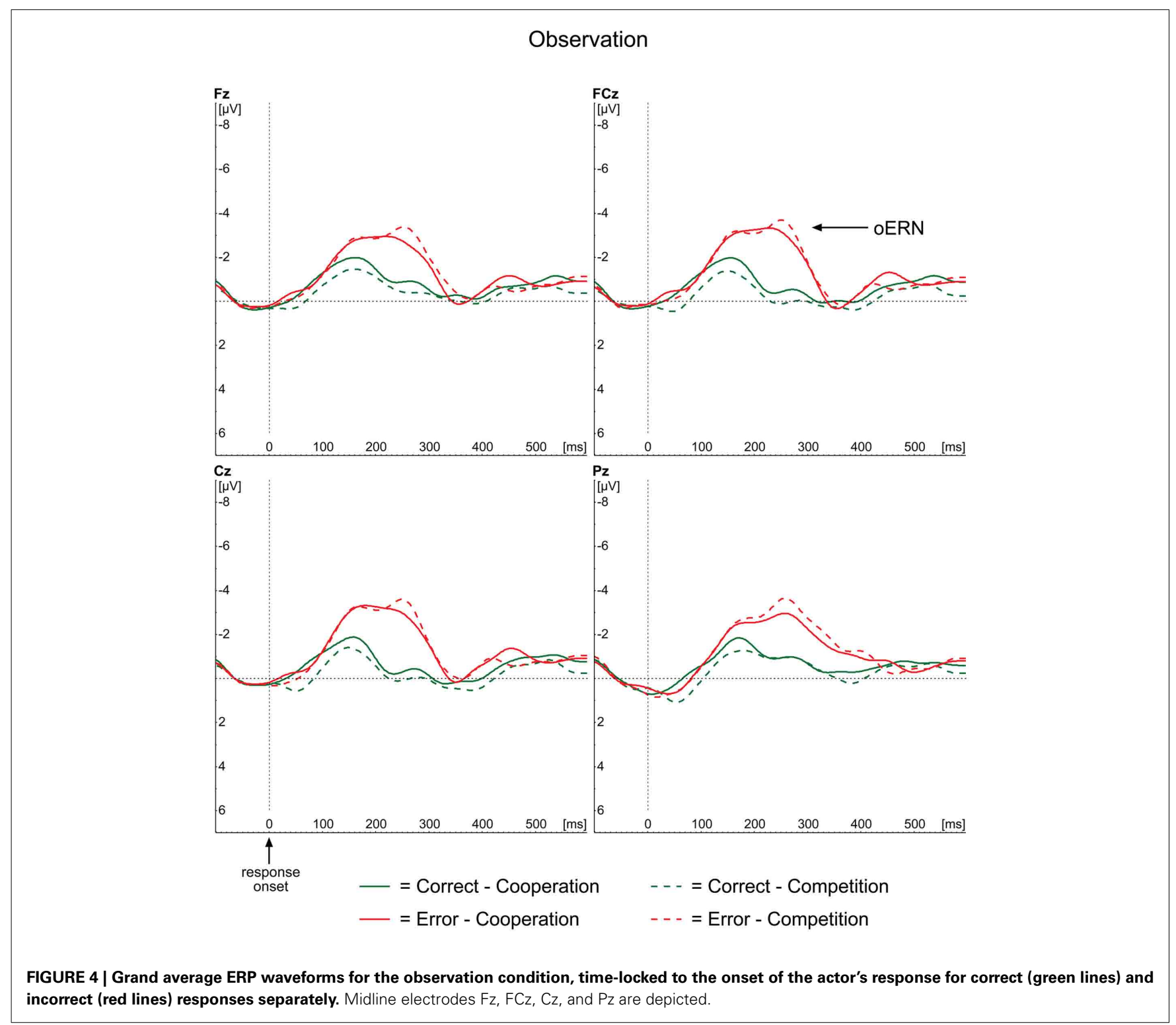

as the stimulus-locked N200, a standard visual ERP component that is typically present in all stimulus-locked ERP waveforms. The important consequence of this interpretation is that the FRN is not - as generally assumed - additionally elicited on erroneous trials, but is always present and may be reduced or even absent on correct feedback trials. This more parsimonious interpretation may explain, for example, why the FRN is also commonly found on non-informative neutral feedback stimuli. Thus, the crucial conclusion was that "events that fail to indicate that a task goal has been achieved (including the occurrence of both neutral and error feedback stimuli) elicit the FRN (or N200), whereas events that do indicate that a task goal has been achieved elicit a positive ERP component," the so-called feedback correct-related positivity or fCRP, significantly reducing or even canceling out the FRN/N200 (Holroyd et al., 2008). The consequence of this interpretation is that the important neural activity is actually happening on correct feedback trials that signal that a goal has been achieved. Although this interesting proposal needs further investigation, it may explain the recent FRN findings in cooperative and competitive contexts. When participants focus on winning in a gambling task, this goal will be achieved when an opponent receives negative feedback. This "negative" feedback signal may thus result in increased positive amplitudes as reflected in the fCRP, importantly reducing the FRN. This alternative interpretation of the FRN may thus explain the reported opposite patterns of FRNs in cooperative and competitive settings using gambling tasks.

It is obvious that future research is needed to test these theories and hypotheses by investigating differences and possible overlap between these ERP components in social settings with more directly comparable experimental paradigms. Instead of using gambling tasks, employing for example RL or probabilistic learning paradigms may be a way to accomplish this, as participants crucially have to use the information derived from the feedback to optimize their behavior. 
A limitation of the current study may be that the blocked observation design does not allow for measurements on behavioral adjustments following observed errors. However, we do believe that the current results may also be relevant in the light of recent behavioral work demonstrating different behavioral adjustments following observed errors in cooperative and competitive contexts (De Bruijn et al., 2011; Núñez Castellar et al., 2011). Post-error slowing, i.e., slowing down following an erroneous response, is a well-known strategic adjustment in speeded-choice-reaction tasks (Rabbitt, 1966). It has repeatedly been shown in individual choicereaction paradigms like the Flankers task, but recent studies have also demonstrated slowing down following other's errors in more social settings (Schuch and Tipper, 2007; De Bruijn et al., 2011; Núñez Castellar et al., 2011). While Shuch and Tipper demonstrated post-error slowing in response to other's errors in a neutral setting, we recently showed that the amount of post-error slowing in response to observed errors critically depends on the context in which the interaction is taking place (De Bruijn et al., 2011). In this behavioral study, we measured both post-error slowing following own and observed errors in both contexts. The results of the cooperative context showed a clear relationship between own and observed post-error slowing: people who slowed down the most following their own errors also slowed down following their partner's errors. However, a different pattern was found in the competitive context. Participants still slowed down following their own errors, but they actually speeded up following errors from their opponents. Núñez Castellar and co-workers recently reported a comparable finding by demonstrating reduced posterror slowing following a co-actor's errors in the competitive context compared to the cooperative one. Based on the previous fMRI study from our lab, we concluded that the findings from our behavioral study indicated that although the error signal itself is insensitive to the context of the social interaction,

\section{REFERENCES}

Bates, A. T., Patel, T. P., and Liddle, P. F. (2005). External behavior monitoring mirrors internal behavior monitoring: error-related negativity for observed errors. J. Psychophysiol. 19, 281-288.

Botvinick, M. M., Cohen, J. D., and Carter, C. S. (2004). Conflict monitoring and anterior cingulate cortex: an update. Trends Cogn. Sci. (Regul. Ed.) 8, 539-546.

Brown, J. W., and Braver, T. S. (2005). Learned predictions of error likelihood in the anterior cingulate cortex. Science 307, 1118-1121.

De Bruijn, E. R. A., de Lange, F. P., von Cramon, D. Y., and Ullsperger, M. (2009). When errors are rewarding. J. Neurosci. 29, 12183-12186.

De Bruijn, E. R. A., Mars, R. B., Bekkering, H., and Coles, M. G. H. (2011). Your mistake is my mistake... or is it? Behavioral adjustments following own and observed actions in cooperative and competitive contexts. Q. J. Exp. Psychol. (Hove). (in press).

the exact formalization of the behavioral adjustments are largely dependent on the context. The current ERP findings are in line with this interpretation, by demonstrating the insensitivity of the error signal to the social context at an electrophysiological level.

To conclude, the present results demonstrate that monitoring other's errors that are time-locked to the actor's response is independent of the reward associated with the observed error. This outcome does not fit with a strict interpretation of the RL theory stating that the ERN should only be generated after a loss of reward. However, we suggest the current findings to be more in line with theories assuming pMFC to be crucially involved in predicting outcomes and adjusting behavior (Rushworth and Behrens, 2008). In our view, the current findings may also be relevant in the light of recent behavioral work demonstrating the social contextspecificity of the exact formalization of behavioral adjustments following observed errors (De Bruijn et al., 2011; Núñez Castellar et al., 2011). Both the context-insensitivity of the error signal and the context-sensitivity for the following behavioral responses make sense in daily life, as it is obvious that different actions may be required in response to an error from one's partner than in response to an error made by an opponent. The current study thus adds further support to the assumption that humans use the same neural and cognitive mechanisms to detect observed errors independent of the associated reward. However, we do propose that the type of behavioral adjustments in response to those observed errors might importantly depend on the role of the co-actor as determined by the social context.

\section{ACKNOWLEDGMENTS}

Ellen R. A. de Bruijn was supported by a VENI grant from the Netherlands Organisation for Scientific Research (NWO; 451-07-022).

Gehring, W. J., Goss, B., Coles, M. G. H., Meyer, D. E., and Donchin, E. (1993). A neural system for error detection and compensation. Psychol. Sci. 4, 385-390.

Gratton, G., Coles, M. G. H., and Donchin, E. (1983). A new method for off-line removal of ocular artifact. Electroencephalogr. Clin. Neurophysiol. 55, 468-484.

Hajcak, G., Holroyd, C. B., Moser J. S., and Simons, R. F. (2005). Brain potentials associated with expected and unexpected good and bad outcomes. Psychophysiology 24, 161-170.

Holroyd, C. B., and Coles, M. G. H. (2002). The neural basis of human error processing: reinforcement learning, dopamine, and the error-related negativity. Psychol. Rev. 109, 679-709.

Holroyd, C. B., Nieuwenhuis, S., Yeung, N., Nystrom, L., Mars, R. B., Coles, M. G. H., and Cohen, J. D. (2004). Dorsal anterior cingulate cortex shows fMRI response to internal and external error signals. Nat. Neurosci. 7, 497-498.

Holroyd, C. B., Pakzad-Vaezi, K. L., and Krigolson, O. E. (2008) The feedback correct-related positivity: sensitivity of the event-related brain potential to unexpected positive feedback. Psychophysiology 45, 688-697.

Itagaki, S., and Katayama, J. (2008). Self-relevant criteria determine the evaluation of outcomes induced by others. Neuroreport 19 , 383-387.

Koban, L., Pourtois, G., Vocat, R., and Vuilleumier, P. (2010). When your errors make me lose or win: event-related potentials to observed errors of cooperators and competitors. Soc. Neurosci. 5, 360-374.

Marco-Pallares, J., Kramer, U., Strehl, S., Schroder, A., and Munte, T. (2010). When decisions of others matter to me: an electrophysiological analysis. BMC Neurosci. 11, 86. doi:10.1186/1471-2202-11-86 
Mars, R. B., Coles, M. G. H., Grol, M. J., Holroyd, C. B., Nieuwenhuis, S., Hulstijn, W., and Toni, I. (2005). Neural dynamics of error processing in medial frontal cortex. Neuroimage 28, 1007-1013.

Miltner, W. H. R., Brauer, J., Hecht, H., Trippe, R., and Coles, M. G. H. (2004). "Parallel brain activity for self-generated and observed errors," in Errors, Conflicts, and the Brain: Current Opinions on Performance Monitoring, eds M. Ullsperger and M. Falkenstein (Leipzig: MPI of Cognitive Neuroscience), 124-129.

Núñez Castellar, E., Notebaert, W., Van den Bossche, L., and Fias, W. (2011). How monitoring other's actions influences one's own performance. Exp. Psychol. 58, 499-508.

Rabbitt, P. M. (1966). Errors and error correction in choice -response tasks. J. Exp. Psychol. 71, 264-272.

Ridderinkhof, K. R., Ullsperger, M., Crone, E. A., and Nieuwenhuis, S. (2004). The role of the medial frontal cortex in cognitive control. Science 306, 443-447.

Rushworth, M. F., and Behrens, T. E. (2008). Choice, uncertainty and value in pre-frontal and cingulate cortex. Nat. Neurosci. 11, 389-397.

Schuch, S., and Tipper, S. P. (2007). On observing another person's actions: influences of observed inhibition and errors. Percept. Psychophys. 69, 828-837.

Shane, M. S., Stevens, M., Harenski, C. L., and Kiehl, K. A. (2008). Neural correlates of the processing of another's mistakes: a possible underpinning for social and observational learning. Neuroimage 42, 450-459.
Singer, T., Seymour, B., O’Doherty, J., Kaube, H., Dolan, R. J., and Frith, C. D. (2004). Empathy for pain involves the affective but not sensory components of pain. Science 20 1157-1162.

van Schie, H. T., Mars, R. B., Coles, M. G. H., and Bekkering, H. (2004). Modulation of activity in medial frontal and motor cortices during error observation. Nat. Neurosci. 7, 549-554.

Walton, M. E., Devlin, J. T., and Rushworth, M. F. (2004). Interactions between decision making and performance monitoring within prefrontal cortex. Nat. Neurosci. 7 1259-1265.

Conflict of Interest Statement: The authors declare that the research was conducted in the absence of any commercial or financial relationships that could be construed as a potential conflict of interest.

Received: 01 December 2011; paper pending published: 16 December 2011; accepted: 15 January 2012; published online: 03 February 2012.

Citation: de Bruijn ERA and von Rhein DT (2012) Is your error my concern? An event-related potential study on own and observed error detection in cooperation and competition. Front. Neurosci. 6:8. doi: 10.3389/fnins.2012.00008

This article was submitted to Frontiers in Decision Neuroscience, a specialty of Frontiers in Neuroscience.

Copyright (C) 2012 de Bruijn and von Rhein. This is an open-access article distributed under the terms of the Creative Commons Attribution Non Commercial License, which permits noncommercial use, distribution, and reproduction in other forums, provided the original authors and source are credited. 